\title{
VIH et allaitement maternel
}

\author{
Jane Helen Downs ${ }^{\mathrm{a}}$ Peter A. Cooper ${ }^{\mathrm{b}}$

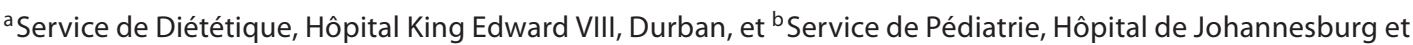 \\ Université du Witwatersrand, Johannesburg, Afrique du Sud
}

\section{Mots-clés}

Transmission mère-enfant $\cdot$ Lait maternel $\cdot$ Mortalité infantile $\cdot$ Mortalité maternelle $\cdot$ Traitement antirétroviral

\section{Résumé}

Il est bien établi que plus de $90 \%$ des enfants infectés par le VIH ont contracté ce virus par transmission mère-enfant (TME). Selon des estimations, 750000 enfants sont infectés par le VIH chaque année au plan mondial et la plupart d'entre eux vivent en Afrique sub-saharienne. La TME se fait au cours de la grossesse par voie transplacentaire, lors de l'accouchement et par l'intermédiaire du lait et de saignements mamelonnaires au cours de l'allaitement. Le risque de TME exprimé en pourcentage varie selon chacune de ces voies. En l'absence d'intervention spécifique, le taux de TME est d'environ $15-20 \%$, et double à $35-40 \%$ quand l'allaitement maternel est prolongé ( $>6$ mois). L'utilisation de substituts du lait maternel (SLM) peut sembler être le choix évident afin de réduire le risque de TME par l'intermédiaire du lait maternel et des saignements mamelonnaires, mais cette option peut s'avérer nocive pour les nourrissons nés de mères vivant dans des contextes où les ressources sont limitées. Dans ces contextes, le risque élevé de mortalité infantile est dû à des diarrhées sévères et à une malnutrition liée à une alimentation peu sûre par SLM et à un allaitement maternel non optimal (allaitement non exclusif pendant les 6 premiers mois de la vie). Selon des estimations présentées dans un rapport récent de l'OMS (2006), 1,45 million de vies d'enfants de moins de 2 ans sont globalement perdues chaque année dans les pays en développement en raison d'un allaitement maternel non optimal, comparativement à 242000 décès de nourrissons liés à la TME. Les recommandations de l'OMS concernant l'alimentation des nourrissons dans le contexte de l'infection à $\mathrm{VIH}$ sont un ensemble important de principes fondamentaux que les gouvernements, les décisionnaires politiques et les profession-

\section{KARGER}

Fax +4161306 1234

E-Mail karger@karger.ch

www.karger.com (c) 2007 Nestec Ltd., Vevey/S. Karger AG, Basel

0250-9644/07/0651-0029\$23.50/0

Accessible en ligne à:

www.karger.com/anf nels de santé doivent prendre en compte pour la conception de politiques et de protocoles de prévention de la TME. Dans les pays tant industrialisés qu'en développement, des communautés ont ou non accès à de l'eau non contaminée et à l'électricité. De ce fait, les politiques doivent répondre aux besoins de communautés différentes. Comme l'indiquent les recommandations de I'OMS: «quand une alimentation de remplacement est acceptable, faisable, financièrement abordable, durable et sûre, l'évitement de tout allaitement par les mères infectées par le VIH est acceptable». Les infections à VIH transmises de façon verticale ont été quasiment éliminées dans les pays industrialisés par l'utilisation appropriée du traitement antirétroviral, la pratique et la programmation d'une césarienne élective et un soutien pour l'évitement de l'allaitement, résultant en une réduction du taux de TME à moins de 1-2\%.

Copyright $\odot 2007$ Nestec Ltd., Vevey/S. Karger AG, Basel

\section{Introduction}

Il est bien établi que plus de $90 \%$ des enfants infectés par le VIH ont contracté ce virus par transmission mère-enfant (TME) [1]. Selon des estimations, 750000 enfants sont infectés par le VIH chaque année au plan mondial et la plupart d'entre eux vivent en Afrique sub-saharienne. La TME se fait au cours de la grossesse par voie transplacentaire, lors de l'accouchement et par l'intermédiaire du lait et de saignements mamelonnaires au cours de l'allaitement. Le risque de TME exprimé en pourcentage varie selon chacune de ces voies. En l'absence d'intervention spécifique, le taux de TME est d'environ 15$20 \%$, et double à 35-40\% quand l'allaitement maternel est prolongé ( $>6$ mois) [2].

L'utilisation de substituts du lait maternel (SLM) peut sembler être le choix évident afin de réduire le risque de TME par l'intermédiaire du lait maternel et des saignements mamelonnaires, mais cette option peut s'avérer nocive pour les nourris-
J.H. Downs

PO Box 50128, Musgrave

Durban, KwaZulu Natal, 4062 (South Africa)

Tel. +27 313603 293, Fax +2731 2016442

E-Mail jane.downs@kznhealth.gov.za 
Tableau 1. Facteurs influençant la décision de choisir des SLM ou l'allaitement maternel

- Droits de l'homme

- Génotype viral et recombinaison entre sous-types chez la mère

- Charge virale et nombre de lymphocytes CD4 chez la mère

- État général et nutritionnel maternel au cours de la grossesse et de l'allaitement

- Facteurs culturels

- Décès de la mère peu après l'accouchement

- Facteurs psychologiques

- Politiques gouvernementales

- Formation et disponibilité insuffisantes de conseillers permettant d'informer les mères tant avant qu'après la naissance

- Impossibilité d'instauration précoce d'un traitement antirétroviral hautement actif chez les mères dont le nombre de lymphocytes CD4 est <200 à la $25^{\text {ème }}$ semaine de grossesse, en raison de ressources humaines trop limitées et d'équipements insuffisants pour traiter les résultats de la numération de CD4

- Mise en œuvre insuffisante de programmes de prévention de la TME

sons nés de mères vivant dans des contextes où les ressources sont limitées. Dans ces contextes, le risque élevé de mortalité infantile est dû à des diarrhées sévères et à une malnutrition liée à une alimentation peu sûre par SLM et à un allaitement maternel non optimal (allaitement non exclusif pendant les 6 premiers mois de la vie). Selon des estimations présentées dans un rapport récent de l'OMS (2006), 1,45 million de vies d'enfants de moins de 2 ans sont globalement perdues chaque année dans les pays en développement en raison d'un allaitement maternel non optimal, comparativement à 242000 décès de nourrissons liés à la TME [3].

La décision de choisir les SLM ou l'allaitement maternel avec le risque de TME qu' il implique est encore compliquée par d'autres facteurs influençant mentionnés dans le tableau 1.

Les recommandations de l'OMS concernant l'alimentation des nourrissons dans le contexte de l'infection à VIH sont un ensemble important de principes fondamentaux que les gouvernements, les décisionnaires politiques et les professionnels de santé doivent prendre en compte pour la conception de politiques et de protocoles de prévention de la TME. Dans les pays tant industrialisés qu'en développement, des communautés ont ou non accès à de l'eau non contaminée et à l'électricité. De ce fait, les politiques doivent répondre aux besoins de communautés différentes. Comme l'indiquent les recommandations de l'OMS: 'quand une alimentation de remplacement est acceptable, faisable, financièrement abordable, durable et sûre, l'évitement de tout allaitement par les mères infectées par le VIH est acceptable' [3].

Les infections à VIH transmises de façon verticale ont été quasiment éliminées dans les pays industrialisés par l'utilisation appropriée du traitement antirétroviral, la pratique et la programmation d'une césarienne élective et un soutien pour l'évitement de l'allaitement, résultant en une réduction du taux de TME à moins de $1-2 \%[4]$.

\section{Droits de l'homme}

Quel que soit son degré d'instruction et d'alphabétisation, toute mère dispose du droit de connaître les bénéfices et risques potentiels associés tant à l'alimentation par SLM qu'à l'allaitement maternel. Dans une société authentiquement démocratique, la mère doit également avoir le droit de choisir les modalités d'alimentation de son enfant. Dans l'idéal, le père $\mathrm{du}$ nourrisson doit intervenir dans ce processus [Downs et Cooper, observations personnelles].

\section{Génotype viral maternel et recombinaison entre sous-types}

Le génotype viral et une recombinaison entre sous-types peuvent influencer le taux et/ou le moment de la TME du VIH-1. Une étude menée en Tanzanie a exploré ce dernier point chez des mères résidant à Dar es Salaam et dont la majorité était atteinte d'une infection à VIH recombinant $(41,0 \%)$, suivi du sous-type A $(26,2 \%)$ ou du sous-type C $(13,1 \%)$ du VIH-1. Une analyse multivariée prenant en compte le nombre des lymphocytes $\mathrm{CD} 4$ de la mère, le stade de l'infection à VIH et la charge provirale dans le lait maternel a montré que le risque de transmission par le lait était 7,2 fois plus élevé quand la mère était infectée par une souche à génome issu d'une recombinaison entre sous-types que lorsqu'elle l'était par un virus de sous-type $C(p=0,02)$. Les virus à longues répétitions terminales (LTR) recombinantes ont été transmis 4,9 fois plus souvent par l'intermédiaire de l'allaitement maternel que ceux à LTR non recombinantes et de sous-type $\mathrm{A}, \mathrm{C}$ ou $\mathrm{D}$. Ces résultats suggèrent que les génomes issus d'une recombinaison entre sous-types, et particulièrement une recombinaison dans les LTR, pourraient rendre le VIH-1 mieux adapté à une transmission par le lait maternel comparativement aux sous-types non recombinants $\mathrm{A}, \mathrm{C}$ et $\mathrm{D}[5]$.

\section{Charge virale et numération de lymphocytes CD4 chez la mère}

Un progrès très important pour la prévention de la TME ces 10 dernières années a été la reconnaissance de l'importance de l'instauration d'un traitement antirétroviral hautement actif (HAART) chez les mères présentant un nombre de lymphocytes $\mathrm{CD} 4<200$ à la $25^{\text {ème }}$ semaine de grossesse. Cette pratique entraîne non seulement une réduction significative de la charge virale, rendant la naissance par voie basse plus sûre (particulièrement dans les pays dont les ressources sont limitées et 
où une césarienne ne peut être effectuée chez toute femme enceinte positive pour le VIH), mais diminue de plus la charge virale dans le lait maternel, réduisant ainsi le risque de TME dans le cas des mères qui choisissent d'allaiter leur nourrisson au cours des 4 à 6 mois suivant sa naissance.

La charge virale du lait maternel varie au cours des 14 premières semaines suivant l'accouchement. Elle a été plus élevée chez des mères présentant une mastite infraclinique et une immunodépression sévère (nombre de lymphocytes CD4 < $200 \times 10^{6}$ cellules/l) [6].

Il est intéressant de noter qu'une étude a indiqué que la charge virale acellulaire dans le lait maternel 1, 6 et 14 semaines après l'accouchement variait considérablement entre les seins droit et gauche à tout moment donné. La charge du lait maternel en VIH-1 a été indétectable dans environ un tiers des échantillons [7].

\section{Rôle de la prévention médicamenteuse chez la mère et le nourrisson}

Un consensus international semble exister selon lequel les femmes enceintes infectées par le VIH et présentant un nombre de lymphocytes CD $4<200$ cellules $/ \mathrm{mm}^{3}$ (ou atteintes d'une infection de stade clinique 3 ou 4 de l'OMS et présentant un nombre de lymphocytes CD $4<350$ cellules $/ \mathrm{mm}^{3}$ ), doivent débuter un HAART à la $25^{\text {ème }}$ semaine de la grossesse ou même au cours du premier trimestre de celle-ci (les bénéfices surpassent les risques de tératogénicité) [8]. Les femmes infectées par le VIH et dont le nombre de lymphocytes CD4 est >200 (et atteintes d'une infection au stade clinique 1 et 2 de l'OMS) doivent recevoir un bref traitement par zidovudine (ZVD) + lamivudine (3-TC) plutôt qu'un seul antirétroviral (par exemple névirapine (NVP) ou ZVD) [9]. Il est important de noter que le risque de résistance à l'HAART chez les mères qui débutent ce traitement dans les 6 mois suivant la prise d'une dose unique de NVP [8] est de plus en plus préoccupant. Les responsables des politiques de santé pourraient donc devoir réviser leurs protocoles de façon correspondante.

L'administration d'un traitement antirétroviral préventif n'est pas unanimement acceptée. Des préoccupations récentes concernant l'hématopoïèse, la prématurité et des anomalies mitochondriales chez des nourrissons et enfants exposés au traitement antirétroviral ont entraîné la nécessité de la prise en compte des risques et bénéfices potentiels de ce type prévention [10-12].

L'administration du triméthoprime-sulfaméthoxazole (TMP-SMZ) pour la prévention du VIH chez les nourissons aux femmes enceintes positives pour le VIH a suscité des inquiétudes, notamment quant à un risque éventuel de tératogénicité si ce traitement est administré au cours du premier trimestre de la grossesse et quant au potentiel d'induction d'une hyperbilirubinémie néonatale et/ou d'un déplacement de la bilirubine de ses sites de liaison à l'albumine s'il est administré à des femmes enceintes proches du terme et au début de la pé- riode d'allaitement. Forna et coll. [13] ont effectué une analyse systématique des données concernant la toxicité du traitement préventif par TMP-SMZ chez les femmes enceintes et allaitantes afin de faciliter l'orientation des pratiques dans des contextes où les ressources sont limitées. Les résultats ont indiqué que la plupart des études analysées avaient démontré que le TMPSMZ n'avait pas été associé à une hyperbilirubinémie quand il avait été administré chez des femmes enceintes ou allaitantes. Aucun cas d'ictère nucléaire n'a été rapporté chez des nouveaunés après traitement des mères par un sulfamide. Les études ont toutefois donné des résultats variables quant au lien entre la prise de TMP-SMZ et d'autres sulfamides au début de la grossesse et un accroissement du risque de fentes palatines, de malformations du tube neural et d'anomalies cardiovasculaires et urinaires. Certaines études ont indiqué qu'une supplémentation en acide folique pouvait faciliter la réduction de certains de ces risques potentiels. En résumé, des données indiquent que le risque de lésions graves du nouveau-né dues à la prise quotidienne d'un traitement préventif par TMP-SMZ par la mère au cours de la grossesse et de l'allaitement est faible. En raison des bénéfices substantiels du traitement préventif par TMP-SMZ des femmes positives pour le VIH vivant dans des contextes où les ressources sont limitées, cette analyse indique qu'il est sûr de suivre les recommandations de l'OMS préconisant l'administration quotidienne de ce traitement chez ces femmes.

Un essai clinique randomisé mené au Botswana de mars 2001 à octobre 2003, appelé étude «Mashi», a évalué l'efficacité et la tolérance de l'allaitement maternel plus prévention par la ZVD chez le nourrisson pendant 6 mois comparativement à celles d'une alimentation par formule plus ZVD pendant 1 mois chez le nourrisson dans la réduction du risque de TME. Dans cette étude, 1200 femmes enceintes infectées par le VIH ont été aléatoirement affectées à un groupe dans 4 hôpitaux de district. Les nourrissons ont été examinés à la naissance, puis mensuellement jusqu'à l'âge de 7 mois, puis à l'âge de 9 mois, et tous les 3 mois jusqu'à l'âge de 18 mois. Toutes les mères ont reçu $300 \mathrm{mg}$ de ZVD par voie orale deux fois par jour à partir de la $34^{\text {ème }}$ semaine de grossesse et au cours du travail. Les mères et les nourrissons ont été aléatoirement affectés à une dose unique de NVP ou à un placebo. Les nourrissons $(\mathrm{n}=1179)$ ont été affectés par randomisation à l'allaitement maternel pendant 6 mois plus ZVD préventive ou à une alimentation par formule plus traitement par ZVD pendant 1 mois. A 7 mois, le taux d'infection à VIH était de 5,6\% chez les nourrissons alimentés par formule et de 9,0\% chez ceux allaités au sein. La mortalité cumulée des nourrissons a cependant été plus élevée dans le groupe alimenté par formule (9,3\%) que dans le groupe allaité au sein (4,9\%). A 18 mois, les taux cumulés de mortalité et d'infection à VIH n'ont pas significativement différé entre les deux groupes (13,9\% dans le groupe alimenté par formule et $15,1 \%$ dans le groupe allaité au sein). Ces résultats soulignent les risques de l'alimentation par formule et les difficultés du choix du mode d'alimentation pour les mères vivant en Afrique sub-saharienne ainsi que la néces- 
sité d'effectuer de nouvelles recherches sur d'autres stratégies. L'instauration d'un HAART chez les femmes enceintes dont le compte des lymphocytes CD4 était <200 à 25 semaines de grossesse peut avoir également réduit le risque de transmission du VIH et de décès chez les nourrissons [14].

Un anti-paludéen, la chloroquine, est actif sur le VIH. En Zambie, l'effet de deux anti-paludéens, la chloroquine et la sulfadoxine-pyriméthamine, sur le taux d'ARN du VIH dans le lait maternel a été évalué chez 30 femmes allaitantes infectées par ce virus. Après correction en fonction du compte de lymphocytes CD4 et de la charge plasmatique virale, la chloroquine a été associée à une tendance à des taux plus faibles d'ARN du VIH dans le lait comparativement à la sulfadoxinepyriméthamine. De nouvelles recherches sont nécessaires afin de déterminer le rôle potentiel de la chloroquine dans la prévention de la TME par l'intermédiaire de l'allaitement maternel [15].

\section{Vaccination des nourrissons}

Un calendrier de vaccination des nourrissons à partir de la naissance faciliterait non seulement la prévention de la TME mais protégerait également les adolescents au début de leur vie sexuelle. Il faudra cependant longtemps avant que cette pratique ne devienne une réalité. Des chercheurs de divers pays travaillent activement au développement d'un vaccin sûr pour les nourrissons [16].

\section{Statut nutritionnel maternel au cours de la grossesse et de l'allaitement}

Un statut nutritionnel adéquat pourrait réduire la fréquence de la TME en réduisant plusieurs facteurs de risque auxquels sont exposés la mère, le fœtus et le nourrisson, notamment par une amélioration de la fonction immunitaire systémique chez la mère ou le fotus/nourrisson, une réduction de la progression clinique, immunologique ou virale chez la mère, une diminution de la charge virale ou du risque d'excrétion virale dans les sécrétions génitales ou le lait, une réduction du risque de prématurité et de faible poids de naissance et un maintien de l'intégrité des voies digestives du fœetus ou de l'enfant. Des études prospectives observationnelles ont indiqué qu'une faible concentration sérique de vitamine A était associée à un accroissement du risque de TME, mais des essais contrôlés et randomisés n'ont pas indiqué que l'administration de cette vitamine ou d'autres avait exercé un effet significatif sur la transmission verticale au cours de la grossesse ou de l'accouchement [17]. Une analyse approfondie d'essais d'interventions par micronutriments chez des femmes enceintes et allaitantes afin d'influencer la croissance, la santé et le statut de leur nouveauné vis-à-vis du VIH est publiée dans le présent numéro des Annales Nestlé [18].
Peu de données sont disponibles quant à l'effet de l'allaitement sur le statut nutritionnel des femmes infectées par le VIH. Une étude récemment publiée de Papathakis et coll. [19] a évalué les modifications de la composition corporelle au cours de l'allaitement chez des femmes infectées ou non par le VIH en Afrique du Sud. Des mesures ont été effectuées à 8 et 24 semaines post-partum chez 92 femmes allaitantes infectées par le VIH et 50 autres non infectées par ce virus. Parmi les femmes allaitantes infectées par le VIH, 95\% présentaient un nombre de lymphocytes CD4 >200 cellules $/ \mathrm{mm}^{3}$. Huit semaines après l'accouchement, la taille, le poids, l'indice de masse corporelle et la masse non grasse des 2 groupes de femmes ne différaient pas significativement. Cependant, 24 semaines après l'accouchement, les mères infectées par le VIH avaient perdu en moyenne $1,4 \mathrm{~kg}$, contre un gain de poids de $0,4 \mathrm{~kg}$ chez celles non infectées. Chez les femmes allaitantes infectées par le VIH, le poids des tissus adipeux sous-cutanés a diminué mais la masse non grasse s'est maintenue.

L'influence de l'allaitement maternel sur l'état de santé de mères infectées par le VIH et de leurs enfants a été évaluée au Malawi (Afrique sub-saharienne) d'avril 2000 à mars 2003. Les mères ont été incluses au moment de la naissance de leur enfant et se sont présentées pour des examens de suivi quand l'enfant était âgé de 1 semaine, 6-8 semaines, puis de 3, 6, 9, 15, 18, 21 et 24 mois. Au total, 2000 femmes infectées par le VIH ont été incluses dans l'étude. Au cours des deux années de l'étude, 44 $(2,2 \%)$ mères et $310(15,5 \%)$ enfants sont décédés. La durée médiane de l'allaitement maternel a été de 18 mois, celle de l'allaitement exclusif de 2 mois et celle de l'alimentation mixte de 12 mois. Après correction en fonction de la charge virale maternelle et de covariables, les types d'allaitement n'ont pas été significativement associés à la morbidité ou la mortalité maternelle. L'allaitement maternel a été associé à une diminution de la mortalité chez les nourrissons et les enfants; le rapport corrigé de risque a été de 0,44 pour l'allaitement maternel global, de 0,45 pour l'alimentation mixte et de 0,40 pour l'allaitement exclusif. Ces derniers effets protecteurs ont été observés chez les nourrissons infectés ou non par le VIH [20].

\section{Durée de l'allaitement maternel et alimentation mixte et SLM}

Une transmission par le lait maternel peut survenir à tout moment de l'allaitement et la probabilité cumulée d'acquisition de l'infection augmente avec la durée de celui-ci [21].

Lors du seul essai randomisé d'intervention publié à ce jour, la morbidité et la mortalité de nourrissons allaités au sein ou alimentés par formule nés de femmes infectées par le VIH-1 ont été évaluées dans quatre centres de traitement prénataux à Nairobi (Kenya). Les mères ont été aléatoirement affectées à une alimentation par formule $(n=186)$ ou à l'allaitement $(\mathrm{n}=185)$ et les nourrissons $(\mathrm{n}=371)$ ont été suivis à 12 et 24 mois. Les formules n'ont pas été fournies gratuitement aux mères lors de cette étude. Le nombre d'enfants en vie et exempts 
d'infection à VIH à 2 ans a été significativement plus bas dans le groupe allaitement maternel que dans le groupe formule. $\mathrm{La}$ proportion cumulée d'infection à VIH à 2 ans de suivi a été de $21 \%$ dans le groupe formule et de $37 \%$ dans le groupe allaitement maternel $(p=0,001)$. Les auteurs ont conclu quavec une éducation appropriée et un accès à de l'eau non contaminée, l'alimentation par formule pouvait être une alternative sûre à l'allaitement maternel pour les nourrissons nés de mères infectées par le VIH-1 dans des contextes où les ressources sont limitées [22].

Une importante étude menée à Harare, Zimbabwe, a évalué l'effet de l'allaitement exclusif sur la mortalité des nourrissons chez 14110 paires mère/nouveau-né. Parmi celles-ci, 4495 mères étaient infectées par le VIH et 2060 de leurs grossesses ont abouti à une naissance vivante. Elles ont été suivies pendant des durées allant jusqu'à 2 ans. Le taux global de TME postnatale a été de 12,1 et $68,2 \%$ de ces transmissions sont survenus après 6 mois, soulignant l'accroissement du risque lié à une prolongation de l'allaitement au-delà du premier semestre. Une alimentation mixte précoce a été associée à un risque plus élevé de TME à 6, 12 et 18 mois [23].

En 2000, Coutsoudis et coll. [24] ont largement attiré l'attention sur le risque potentiel de l'alimentation mixte comparativement à l'allaitement exclusif. Les résultats d'une étude menée à Durban, Afrique du Sud, ayant évalué les pratiques d'alimentation des nourrissons de 549 femmes infectées par le VIH ont indiqué que, vers l'âge de 3 mois, les nourrissons recevant un allaitement maternel exclusif avaient été moins souvent infectés par le VIH $(14,3 \%)$ que ceux recevant une alimentation mixte $(24,1 \%)$ [24].

Une méta-analyse de données ayant trait à la transmission post-natale tardive du VIH-1 chez 4085 nourrissons allaités au sein lors de 9 essais a indiqué que le moment de l'infection était connu chez 539 de ces nourrissons, et que, parmi ceux-ci, 225 (42\%) avaient contracté le virus en période post-natale tardive (allaitement maternel au-delà de 6 mois). La probabilité cumulée de transmission post-natale tardive à 18 mois a été de 9,3\% [25].

La prévalence de l'alimentation mixte (au lieu de l'allaitement maternel exclusif) paraît élevée dans des pays où les ressources sont limitées [26]. Certaines études ont indiqué que de nombreuses femmes instauraient une alimentation mixte en raison de l'insuffisance de leur lait. La décision d'effectuer une alimentation mixte est cependant sans doute prise sur la base de plusieurs facteurs. Une étude longitudinale menée dans une communauté rurale aux ressources limitées et où la prévalence du VIH était élevée (Mtubatuba, KwaZulu Natal, Afrique du Sud) a suivi 119 nourrissons pendant 16 semaines. De plus, une enquête transversale a été menée auprès des mères de 445 nourrissons. Les résultats ont montré qu'une faible proportion (10\%) des nourrissons était exclusivement allaitée pendant 6 semaines. Le motif perçu le plus fréquent d'alimentation mixte a été une insuffisance de lait maternel. Une autre observation préoccupante dans l'aspect longitudinal de l'étude a été que $46 \%$ des nourrissons ont reçu des liquides autres que le lait maternel ou une formule dans les 48 heures suivant leur naissance. Le choix de l'alimentation par la mère a été principalement autodéterminé (43\%), tandis que des travailleurs sociaux (22\%) et la grand-mère (16\%) ont été cités comme les principales sources de conseil [27]. Ces résultats permettent d'en déduire la nécessité d'une formation plus efficace des travailleurs sociaux et de campagnes de promotion ciblant les femmes dans les contextes où les ressources sont limitées et portant sur l'importance de l'alimentation maternelle exclusive pendant les 4-6 premiers mois.

Une étude menée en Afrique du Sud [28] a évalué l'influence du mode d'alimentation sur la morbidité infantile à des visites cliniques réalisées à 1 et 6 semaines, à 3 mois puis tous les 3 mois par la suite pendant 15 mois. Les résultats ont montré que le pronostic était plus péjoratif pour les nourrissons infectés par le VIH qui n’avaient jamais été allaités au sein que pour ceux qui l'avaient été. Neuf (60\%) des nourrissons qui n’avaient jamais été allaités ont présenté au moins 3 épisodes morbides comparativement à 15 (32\%) des enfants allaités. Au cours des 2 premiers mois de leur vie, les nourrissons non allaités au sein (quel qu'ait été leur statut vis-à-vis du VIH) ont présenté près de deux fois plus d'épisodes pathologiques que les nourrissons allaités. Ces résultats soulignent le risque de l'alimentation par formules dans des contextes où les ressources sont limitées. Cette étude a été menée avant la mise en œuvre du programme antirétroviral complet sud-africain.

De nouvelles recherches sont nécessaires afin de mieux connaître les motifs pour lesquels les mères choisissent de ne pas effectuer un allaitement exclusif au sein, particulièrement dans les communautés dont les ressources sont limitées. En sus des facteurs susmentionnés, les mères qui travaillent peuvent ne pas avoir un congé de maternité adéquat, ne pas disposer d'installations leur permettant de prendre soin de leur enfant sur leur lieu de travail et travailler de longues heures, autant de facteurs qui rendent très difficiles l'instauration et la poursuite d'un allaitement maternel exclusif. Dans de nombreux pays, aucune législation ne protège l'allaitement maternel sur le lieu de travail. De façon ironique, de nombreux hôpitaux (dont plus des deux tiers du personnel se composent habituellement de femmes) certifiés comme «accueillants pour les bébés» ne disposent pas de locaux dédiés aux soins maternels des nourrissons. De ce fait, les travailleuses hospitalières ne peuvent pas allaiter leurs nourrissons au cours de la journée. Il semble peu raisonnable et peu crédible d'attendre d'une travailleuse de santé quelle promeuve et soutienne l'allaitement maternel si elle-même ne dispose pas de la possibilité d'allaiter sur son lieu de travail [Downs, observation personnelle].

La perméabilité intestinale du nourrisson a été également associée à un risque plus élevé de TME. Une étude au cours de laquelle 272 nourrissons de femmes infectées par le VIH en Afrique du Sud ont été examinés aux âges de 1, 6 et 14 semaines et ont fait l'objet d'un test aux deux sucres lactulose/mannitol a montré que les nourrissons infectés par le VIH au cours des 14 premières semaines présentaient une perméabilité intestinale significativement plus élevée à 6 et 14 semaines et une ex- 
crétion urinaire légèrement plus élevée de la néoptérine (indicateur de l'activation du système immunitaire) à tous les moments de mesure comparativement aux nourrissons non infectés. Le mode d'alimentation n'a exercé aucun effet sur l'excrétion de la néoptérine, un indice de l'immuno-stimulation. Ces résultats suggèrent que l'infection à VIH du nourrisson induit des modifications de la perméabilité intestinale et, peut-être, une activation du système immunitaire avant l'apparition de manifestations cliniques [29].

Si les mères doivent être incitées à cesser l'allaitement maternel à 6 mois, il est nécessaire que les concepteurs de politiques et les travailleurs sociaux s'assurent qu'elles reçoivent des informations et des conseils adéquats sur les aliments complémentaires ou de sevrage [30].

\section{Facteurs culturels influençant les pratiques d'alimentation des nourrissons}

Dans certaines cultures, la grand-mère ou le père de l'enfant décide du mode et de la durée de l'alimentation du nourrisson, et cette décision supplante habituellement tout conseil donné dans un centre de soins.

Une intéressante étude menée au Zimbabwe a porté sur les facteurs influençant le choix du mode d'alimentation du nourrisson dans une population de femmes vivant dans des zones urbaines (200 femmes aux centres cliniques de Harare et Chitungwiza) dans le contexte de la transmission du VIH. Les résultats ont indiqué que l'influence des maris (58\%) sur les pratiques alimentaires avait été plus importante que celle des infirmières (42\%), suggérant que les influences sociales sont plus fortes que l'avis du personnel médical quant au choix d'une méthode d'alimentation. Respectivement 33 et $77 \%$ des femmes de Harare et Chitungwiza connaissaient le lien entre la transmission du VIH et l'allaitement maternel. Quaranteneuf pour-cent des femmes redoutaient d'allaiter. Le degré d'instruction, le statut vis-à-vis de l'emploi et les opinions de la famille ont tous influencé les décisions des femmes enceintes infectées par le VIH quant au mode d'alimentation du nourrisson [31].

Plus de 90\% des 164 mères interrogées lors d'une étude menée dans une zone rurale du Zimbabwe ont cependant indiqué qu'elles avaient pris personnellement la décision d'allaiter leur enfant. Un tiers de ces répondeuses ont également mentionné qu'elles avaient pris en compte les messages des travailleurs sociaux. Les chercheurs ont recommandé d'accroître le soutien alimentaire des nourrissons dans les populations rurales à ressources limitées. Ce soutien doit être envisagé en termes de formation des équipes de conseillers, de suivi décentralisé et de soutien au sevrage [32].

Une étude qualitative longitudinale menée dans trois centres en Afrique du Sud a déterminé les pratiques et processus de prise de décision concernant l'alimentation des nourrissons chez des femmes enceintes positives pour le VIH. Les femmes ont indiqué au cours de la période prénatale si elles avaient l'inten- tion de nourrir exclusivement leur enfant par SLM ou au sein. Un peu moins de 50\% des femmes qui avaient l'intention d'allaiter exclusivement au sein ont maintenu cette exclusivité, tandis que deux tiers de celles qui avaient débuté une alimentation par formule ont maintenu exclusivement ce mode. Les observations ont indiqué que les principales caractéristiques des femmes ayant maintenu l'exclusivité étaient l'aptitude à résister aux pressions de la famille visant à introduire d'autres liquides et à se rappeler des messages clés sur les risques de TME et ceux de l'alimentation mixte. Les femmes ayant maintenu l'allaitement exclusif au sein étaient particulièrement convaincues des bénéfices de celui-ci. Un environnement confortant au domicile s'est également avéré important. Les mères ayant choisi une alimentation exclusive par formule ont mentionné que le fait de disposer de ressources telles que l'électricité, une bouilloire et un flacon facilitait l'alimentation la nuit [33].

\section{Facteurs psychologiques et socioéconomiques}

Les femmes vivant dans des contextes à ressources limitées en Afrique et en Asie sont confrontées à de multiples difficultés, dont une augmentation de la vulnérabilité au VIH et la charge de nourrir et de prendre en charge des orphelins de membres de la famille et d'amis décédés. Si elles sont infectées par le VIH, une autre difficulté est le mode d'alimentation du nourrisson [34].

Les mères vivant dans des conditions de ressources limitées peuvent choisir une alimentation par SLM car redoutant une TME, bien que cette modalité puisse exposer à d'autres risques. Lors d'une étude menée dans des zones semi-rurales situées à la périphérie de Lilongwe au Malawi, 22 femmes infectées par le VIH ont été interrogées quant à leur perception de leur état de santé et de l'effet de l'allaitement au sein sur celuici. Plusieurs d'entre elles ont considéré que leur statut nutritionnel se dégradait en raison de leur maladie. Ces femmes étaient également préoccupées par une possibilité d'accélération de la progression de la maladie sous l'effet de l'allaitement. L'effectif de la population incluse dans l'étude était restreint, mais ces résultats soulignent néanmoins l'importance de conseils très détaillés [35].

Une autre étude a porté sur l'effet du programme de prévention de la TME en Afrique du Sud sur l'alimentation et les pratiques de soins des nourrissons dans une communauté semi-urbaine située à proximité de la ville du Cap (Khayelitsha). Ce programme donnait aux mères infectées par le VIH l'option d'allaiter exclusivement au sein ou d'alimenter exclusivement par formule pendant 6 mois. Les femmes infectées par le VIH ont indiqué qu'elles n'avaient ressenti aucun effet social négatif lié au fait qu'elles n'allaitaient pas [36]. Il peut toutefois ne pas en être de même dans d'autres communautés d'Afrique du Sud, particulièrement dans des zones rurales où l'allaitement maternel est la norme.

Diverses publications ont indiqué que les mères infectées par le VIH considéraient qu'il était difficile d'arrêter subite- 
ment l'allaitement au sein 4 à 6 mois après l'accouchement. Il est bien établi que l'allaitement maternel est non seulement la forme irremplaçable d'alimentation des nourrissons, mais joue également un rôle important dans le développement affectif de l'enfant et pour la féminité de la mère $[37,38]$.

\section{Décès maternel peu après l'accouchement: pasteurisation du lait humain et lactariums}

Le décès de la mère peu après l'accouchement est une difficulté majeure pour les gouvernements et communautés dont les ressources sont limitées. Le risque estimé de mortalité des enfants africains nés de mères infectées par le VIH a été extrapolé dans le cadre d'une analyse de données regroupées (données de 7 essais randomisés d'intervention sur la TME en Afrique). Sur 3468 enfants, 378 (11\%) sont décédés. Les estimations indiquent que $35,2 \%$ des enfants infectés et $4,9 \%$ de ceux non infectés sont décédés en un an. La mortalité infantile a varié en fonction de la région géographique et a été associée au décès des mères [39]. Le type et le moment adéquats de l'instauration d'un HAART peuvent non seulement faciliter la prévention du décès maternel mais également de celui du nourrisson.

Les lactariums ont été presque tous fermés il y a environ 20 ans en raison de préoccupations concernant le risque de transmission du VIH, mais le concept a été réévalué ces dernières années. Le Brésil, en particulier, a constitué un programme étendu de lactariums destinés à fournir du lait de femme à des nourrissons orphelins. Le lait est traité par la chaleur afin d'inactiver le VIH.

L'infection à VIH a contribué à un accroissement significatif de la naissance de prématurés de petit et très petit poids de naissance. Dans ce groupe de nourrissons, l'alimentation par formule a été associée à une augmentation du risque d'entérocolite nécrosante. En conséquence, certaines institutions d'Afrique du Sud (Le Cap, Pretoria et Durban) ont fondé des lactariums afin d'apporter à ces nourrissons du lait de femme traité par la chaleur. Il s'agit également d'une option utile pour les mères atteintes d'une mastite [40].

Israel-Ballard et coll. [41] ont développé une méthode rapide de pasteurisation à domicile, appelée "méthode flash», qui élimine l'activité virale et détruit la contamination bactérienne sans altérer la composition en nutriments. Coutsoudis et coll. [2006, données non publiées] ont évalué cette méthode dans une communauté périurbaine de Durban, Afrique du Sud, et ont constaté qu'elle était une façon pratique et sûre de pasteurisation du lait de femme pour les nourrissons âgés de 6 mois et plus. Il pourrait s'agir d'une méthode précieuse pour les mères vivant dans des contextes à ressources limitées et devant reprendre leur travail dans les 6 semaines suivant l'accouchement, et donc réduire la fréquence de l'alimentation mixte. Cette dernière hypothèse nécessite des études supplémentaires.

VIH et allaitement maternel

\section{Politiques gouvernementales et programmes nationaux de prévention de la TME}

Les politiques gouvernementales de prévention de la TME varient en fonction des ressources disponibles. Les révisions de politiques tendent à être un processus lent et, de ce fait, ne sont souvent pas en accord avec les observations ou données scientifiques les plus récentes. Dans certains pays, des politiques écrites peuvent ne pas être disponibles pour les centres de soins, particulièrement en zone rurale.

Certains pays ont démontré les bénéfices de la mise en œuvre efficace de politiques nationales de prévention entraînant une réduction significative de la TME (taux bas de 1-2\%). Il est important d'auditer la mise en œuvre des politiques et de déterminer si elles sont instaurées de façon optimale et sont efficaces [2].

Une enquête récemment publiée menée en Grande-Bretagne afin d'évaluer l'observance des recommandations 2001 de la British HIV Association concernant la prise en charge des femmes enceintes infectées par le VIH a montré que, en règle générale, ces recommandations avaient été mises en pratique. Les principaux critères évalués étaient l'administration adéquate d'un traitement antirétroviral, la réalisation et le moment d'une césarienne élective et un soutien pour l'évitement de l'allaitement maternel. Sur 186 centres enquêtés au Royaume-Uni, 100 (54\%) ont répondu en communiquant des données sur 501 grossesses éligibles. Les recommandations ont généralement été correctement appliquées, à l'exception d'un certain nombre de césariennes planifiées plus tardivement qu'au moment recommandé, à la $38^{\text {ème }}$ semaine de grossesse [42].

En Australie, l'impact d'une campagne d'éducation sur la prise en charge des femmes enceintes infectées par des virus à transmission sanguine (dont le VIH) a été évalué par 2 enquêtes consécutives en 2002 et 2004. En 2002-2003, lors de la première enquête, 767 membres enregistrés auprès du RANZCOG (Registered Australian and New Zealand College of Obstetricians and Gynaecologists) ont reçu des questionnaires par voie postale et, lors de la seconde enquête en 2004 , les questionnaires ont été expédiés à 743 de ces membres. Après la première enquête, une intervention pluridimensionnelle a eu lieu, comportant l'expédition des résultats de l'enquête et un résumé de la prise en charge recommandée, la publication de deux articles d'analyse dans le journal du RANZCOG et une présentation orale au congrès scientifique annuel du RANZCOG. Lors de la seconde enquête (taux de réponse de 68\%), les répondeurs ont indiqué une augmentation de la fréquence du dépistage de virus à transmission sanguine, des modifications des pratiques sur la base du statut des femmes vis-à-vis de l'infection et des conseils donnés aux femmes enceintes quant au risque de transmission de virus par l'intermédiaire de l'allaitement au sein. En 2004, une augmentation (de 51\% lors de la première enquête à $59 \%$ lors de la seconde) des répondeurs ayant indiqué qu'ils avaient proposé en routine un dépistage anténatal du VIH a été observée. Les résultats ont indiqué une 
augmentation de $12 \%$ (47\% des répondeurs) du nombre des répondeurs ayant systématiquement recommandé aux femmes enceintes infectées par le VIH de choisir une césarienne élective. Ces observations ont démontré que les connaissances sur les interventions destinées à réduire la TME à la naissance et par l'intermédiaire de l'allaitement maternel se sont améliorées après une intervention éducative relativement simple [43].

Dans les pays où résident des communautés à ressources limitées, une simulation mathématique par modélisation peut être un outil utile pour la prévision du risque de TME et de mortalité infantile, afin d'informer sur les décisions politiques, avec référence spécifique au VIH et à l'alimentation des nourrissons [44].

\section{Formation et disponibilité inadéquates de conseillers informant les mères tant avant qu'après l'accouchement}

Dans certains contextes, les conseillers peuvent laisser leurs opinions personnelles, et parfois dogmatiques, prévaloir au lieu de donner des conseils objectifs quant aux avantages et inconvénients de l'alimentation par SLM et de l'allaitement au sein à des mères vivant dans un environnement donné. A Moshi, en Tanzanie du Nord, des conseillers ont été interrogés quant à leurs points de vue sur un test prénatal de détection du VIH et les dilemmes concernant l'alimentation des nourrissons auxquels sont confrontées les femmes infectées par le VIH dans leur communauté [45]. L'expérience de ces conseillers allait de 6 mois à 9 ans. Les résultats ont indiqué que, quel qu'ait été le statut socioéconomique maternel, le choix éclairé d'une méthode d'alimentation du nourrisson par les femmes infectées par le VIH, comme préconisé par les recommandations ONUSIDA/OMS/UNICEF, avait été gravement compromis par l'avis réellement donné, des conseils directifs, le manque de temps pour permettre d'affronter une positivité du test et l'insuffisance de soutien de suivi. Les options d'alimentation du nourrisson n'ont pas toujours été expliquées avec précision, mais les conseillers ont considéré que la plupart des femmes avaient peu d'autre choix que d'allaiter et qu'il était peu probable qu'elles le fassent de façon exclusive malgré les conseils donnés. L'enquête a montré que les risques et bénéfices des options disponibles pour les femmes infectées par le VIH étaient non seulement un sujet complexe pour celles-ci, mais également pour les conseillers. Les auteurs ont recommandé que les conseillers suivent une formation supplémentaire en matière de conseils non directifs et d'options d'alimentation des nourrissons afin d'assurer une meilleure qualité des informations et du soutien de suivi des femmes à leur domicile. Une politique nationale peut exister dans de nombreux pays, mais, fréquemment, la réalisation pratique et la formation sont négligées ou absentes [Downs et Cooper, observation personnelle].

Piwoz et coll. [46] ont évalué les différences entre les recommandations internationales sur l'allaitement maternel dans le contexte de l'infection à VIH et les attitudes et messages de conseil de 19 travailleurs sociaux à Lilongwe, Malawi. Les travailleurs sociaux expérimentés en matière de conseils ont considéré que les mères infectées par le VIH devaient allaiter exclusivement au sein plutôt que d'alimenter leur nourrisson par formule, citant la pauvreté comme principale raison. Tous les travailleurs de santé ont fait part de leurs préoccupations quant à l'arrêt précoce de l'allaitement maternel en raison du degré élevé de malnutrition dans leur communauté.

Les conseils oraux tendent à être la méthode de choix pour l'éducation des mères infectées par le VIH sur les risques et bénéfices des différentes options d'alimentation des nourrissons. Dans un programme mené à Harare, Zimbabwe, et ayant inclus 14110 paires mère/nourrisson, toutes ont été testées pour le VIH mais n'étaient pas dans l'obligation de connaître leur statut vis-à-vis du virus. Le programme a utilisé plusieurs moyens éducatifs dont éducation de groupe, conseils individuels, vidéos et brochures. Il est intéressant de noter que, après le déploiement total du programme, les femmes ayant appris précocement leur statut ont été plus nombreuses de $70 \%$ et ont allaité exclusivement au sein 8,4 fois plus souvent que les mères incluses au moment où le programme débutait [47].

\section{Mise en œuvre médiocre de programmes de prévention de la TME}

Les mères peuvent pouvoir choisir les modalités d'alimentation mais ne reçoivent pas de conseils appropriés quant à une alimentation sûre. Dans certains pays, les mères qui choisissent l'alimentation par SLM ne reçoivent pas d'informations écrites dans leur langue maternelle quant à la préparation sûre et à la dilution correcte de ces préparations, qui leur sont insuffisamment remises. Dans certains pays, par exemple en Afrique du Sud, les mères qui choisissent d'alimenter exclusivement leur nourrisson par SLM ne reçoivent que 6 à 8 boîtes de ces produits par mois, ce qui ne représente qu'environ $50 \%$ des besoins nutritionnels des nourrissons âgés de 4 à 6 mois, ce qui entraîne une tendance des mères à trop diluer ces préparations ou à recourir à une alimentation mixte, augmentant ainsi le risque de TME et d'insuffisance staturo-pondérale du nourrisson. Cette situation est encore compliquée par l'arrêt brutal de la fourniture des SLM quand le nourrisson atteint l'âge de 6 mois. Dans l'idéal, le volume de SLM devrait être progressivement diminué après l'âge de 6 mois et les mères et leurs aidants devraient recevoir des conseils écrits et oraux adéquats quant aux aliments de sevrage [Downs et Cooper, observation personnelle].

De plus, les mères qui choisissent d'allaiter leur nourrisson au sein ne reçoivent pas toujours un soutien adéquat sous forme de conseils dans des centres cliniques et lors de visites à domicile, ni des informations appropriées sur la nutrition de leur nourrisson 6 mois après l'accouchement. Ces mères ne 
sont pas toujours conseillées quant aux méthodes de pasteurisation du lait de femme quand le nourrisson atteint 4-6 mois (les politiques varient d'un pays à l'autre) [Downs et Cooper, observation personnelle].

\section{Recommandations de l'OMS}

Les recommandations de l'OMS ayant trait à la prévention de la TME du VIH et leurs implications politiques, conclusions et préconisations (2001) conseillent: «quand une alimentation de remplacement est acceptable, faisable, financièrement abordable, durable et sûre, l'évitement de tout allaitement par les mères infectées par le VIH est acceptable». Il s'agit d'une importante préconisation pour tous les travailleurs sociaux qui sont en relation avec les femmes enceintes et allaitantes.

Certains auteurs ont fait part de leurs préoccupations en indiquant que, si ces recommandations existent effectivement, elles devraient être renforcées par des investissements dans des actions de conseils sur le VIH de haute qualité et largement disponibles, dans un soutien pour le choix de l'alimentation et pour l'allaitement maternel exclusif pour les mères infectées par le VIH qui choisissent ce mode d'alimentation [48].

Une étude a été menée à Ile-Ife au sud-ouest du Nigeria, où l'allaitement maternel est la norme (mais n'est pas nécessairement exclusif), afin de déterminer l'acceptabilité, la faisabilité, la possibilité financière, la sécurité d'emploi et la durée des options d'alimentation de substitution pour les mères infectées par le VIH. Les résultats ont montré que les obstacles au recours aux aliments de substitution (au lieu de l'allaitement maternel) étaient notamment le coût élevé de ces produits et des combustibles nécessaires pour les cuisiner, la fourniture non fiable d'électricité et un accès très restreint à de l'eau non contaminée et à des moyens de conservation des aliments [49].

\section{Conclusion}

Dans les pays industrialisés, le plus faible risque de TME est lié à une naissance par césarienne, à une prévention antirétrovirale adéquate et à une alimentation exclusive par formule. Dans les communautés dont les ressources sont limitées, l'allaitement au sein et l'alimentation par formules comportent tous deux des risques pour les nourrissons exposés au VIH et l'équilibre des risques varie en fonction des contextes et au cours du temps [50].

En conclusion, l'objectif doit être non seulement de prévenir la TME mais également la mortalité infantile due à d'autres facteurs. De plus, aucun concepteur de politique ou travailleur social (sauf s'il s'est trouvé lui-même dans la même situation) ne comprendra jamais réellement toute l'importance de l'anxiété d'une mère positive pour le VIH et les dilemmes qu'elle doit affronter quand elle doit décider du mode d'alimentation de son nourrisson et, dans certains cas, la culpabilité et la dépression qu'elle ressent quand elle découvre que son enfant a contracté le VIH par TME.

\section{Remerciements}

Sincères remerciements à Nisha Padayachee (diététicien, Hôpital King Edward VIII, Durban) pour sa relecture du présent article.

\section{Références}

1 Luo C: Strategies for prevention of motherto-child transmission of HIV. Reprod Health Matters 2000;8:144-155.

2 Newell ML: Current issues in the prevention of mother-to-child transmission of HIV-1 infection. Trans R Soc Trop Med Hyg 2006; 100:1-5.

3 Lauer JA, Betran AP, Barros AJ, de Onis M: Deaths and years of life lost due to sub-optimal breast-feeding among children in the developing world: a global ecological risk assessment. Public Health Nutr 2006;9:673685.

-4 Thorne C, Newell ML: Treatment options for the prevention of mother-to-child transmission of HIV. Curr Opin Investig Drugs 2005; 6:804-811.
5 Koulinska IN, Villamor E, Msamanga G, et al: Risk of HIV-1 transmission by breastfeeding among mothers infected with recombinant and non-recombinant HIV-1 genotypes. Virus Res 2006;120:191-198.

6 Willumsen JF, Filteau SM, Coutsoudis A, et al: Breastmilk RNA viral load in HIV-infected South African women: effects of subclinical mastitis and infant feeding. AIDS 2003. 17:407-414.

7 Willumsen JF, Newell ML, Filteau SM, et al: Variation in breastmilk HIV-1 viral load in left and right breasts during the first 3 months of lactation. AIDS 2001;15:18961898 .

8 World Health Organization - HIV/AIDS Program: Antiretroviral therapy for HIV infection in adults and adolescents in resourcelimited settings: towards universal access. E-pub 2006;www.who.int/hiv/pub/guidelines/adult/en/index.html.
9 Leroy V, Sakarovitch C, Cortin-Borja M, et al; Ghent Group on HIV in Women and Children: Is there a difference in the efficacy of peripartum antiretroviral regimes in reducing mother-to-child transmission of HIV in Africa? AIDS 2005;19:1865-1875.

10 Thorne C, Newell ML: Prevention of mother-to-child transmission of HIV infection. Curr Opin Infect Dis 2004;17:247-252.

- 11 Thorne C, Newell ML: Antenatal and neonatal antiretroviral therapy in HIV-infected women and their infants: a review of safety issues. Med Wieku Rozwoj 2003;7:425-436.

12 Gaillard P, Fowler MG, Dabis F, et al: Use of antiretroviral drugs to prevent HIV-1 transmission through breast-feeding: from animal studies to randomized clinical trials. J Acquir Immune Defic Syndr 2004;35:178187. 
13 Forna F, McConnell M, Kitabire FN, et al: Systematic review of the safety of trimethoprim-sulfamethoxazole for prophylaxis in HIV-infected pregnant women: implications for resource-limited settings. AIDS Rev 2006;8:24-36.

14 Thior I, Lockman S, Smeaton LM, et al: Breastfeeding plus infant zidovudine prophylaxis for 6 months vs. formula feeding plus infant zidovudine for 1 month to reduce mother-to-child HIV transmission in Botswana: a randomized trial: the Mashi Study. JAMA 2006;296:794-805.

$\checkmark 15$ Semrau K, Kuhn L, Kasonde P, et al: Impact of chloroquine on viral load in breast milk. Trop Med Int Health 2006;11:800-803.

16 Luzuriaga K, Newell ML, Dabis F, et al: Vaccines to prevent transmission of HIV-1 via breastmilk: scientific and logistical priorities. Lancet 2006;368:511-521.

17 Fawzi W: Nutritional factors and vertical transmission of HIV-1. Epidemiology and potential mechanisms. Ann NY Acad Sci 2000;918:99-114.

18 Mehta S, Finklestein JL, Fawzi W: Nutritional interventions in HIV-infected breastfeeding women. Ann Nestlé [Engl] 2006;65:3948.

19 Papathakis PC, Van Loan MD, Rollins NC, et al: Body composition changes during lactation in HIV-infected and HIV-uninfected South African women. J Acquir Immune Defic Syndr 2006;43:467-474.

-20 Taha TE, Kumwenda NI, Hoover DR, et al: The impact of breastfeeding on the health of HIV-positive mothers and their children in sub-Sahara Africa. Bull World Health Organ 2006;84:546-554.

-21 John-Stewart G, Mbori-Ngacha D, Ekpini R, et al; Ghent IAS Working Group on HIV in Women and Children: Breast-feeding and transmission of HIV-1. J Acquir Immune Defic Syndr 2004;35:196-202.

-22 Mbori-Ngacha D, Nduati R, John G, et al: Morbidity and mortality in breastfed and formula fed infants of HIV1-infected women: a randomized clinical trial. JAMA 2001; 286:2413-2420.

23 Iliff PJ, Piwoz EG, Tavengwa NV, et al; ZVITAMBO Study Group: Early exclusive breastfeeding reduces the risk of post-natal HIV-1 transmission and increase HIV-free survival. AIDS 2005;19:699-708.

-24 Coutsoudis A: Influence of infant feeding patterns on early mother-to-child transmission of HIV-1 in Durban, South Africa. Ann NY Acad Sci 2000;918:136-144.

-25 Coutsoudis A, Dabis F, Fawzi W, et al; Breastfeeding and IIV International Transmission Study Group: Late postnatal transmission of HIV-1 in breast-fed children: an individual patient data meta-analysis. J Infect Dis 2004; 189:2154-2166.
26 Ehrnst A, Zetterstrom R: Vertical transmission of HIV-1 infection and dilemma of infant feeding. Acta Paediatr 2003;92:990991.

27 Bland RM, Rollins NC, Coutsoudis A, Coovadia HM; Child Health Group: Breastfeeding practices in an area of high HIV prevalence in rural South Africa. Acta Paediatr 2002;91:704-711.

28 Coutsoudis A, Pillay K, Spooner E, et al: Morbidity in children born to women infected with human immunodeficiency virus in South Africa: does mode of feeding matter. Acta Paediatr 2003;92:890-895.

29 Rollins NC, Filteau SM, Coutsoudis A, Tomkins AM: Feeding mode, intestinal permeability, and neopterin excretion: a longitudinal study in infants of HIV-infected South African women. J Acquir Immune Defic Syndr 2001;28:132-139.

30 Piwoz EG, Huffman SL, Quinn VJ: Promotion and advocacy for improved complimentary feeding: can we apply the lessons learned from breastfeeding? Food Nutr Bull 2004;25: 303-304.

31 Gara CP, Pazvakavambwa I, Maponga CC, Gavaza P: An investigation of the factors influencing the infant feeding methods among urban Zimbabwean women in the context of HIV transmission. Cent Afr J Med 2005;51: $1-4$.

32 Orne-Gliemann J, Mukotekwa T, Miller A, et al: Community-based assessment of infant feeding practices within a programme for prevention of mother-to-child HIV transmission in rural Zimbabwe. Public Health Nutr 2006;9:563-569.

33 Doherty T, Chopra M, Nkonki L, et al: A longitudinal qualitative study of infant-feeding decision making and practices among HIVpositive women in South Africa. J Nutr 2006; 136:2421-2426.

-34 Piwoz EG, Bentley ME: Women's voices, women's choices: the challenge of nutrition and HIV/AIDS. J Nutr 2005;135:933-937.

35 Bentley ME, Corneli AL, Piwoz E, et al: Perceptions of the role of maternal nutrition in HIV-positive breast-feeding women in Malawi. J Nutr 2005;135:945-949.

36 Chopra M, Piwoz E, Sengwana J, et al: Effect of a mother-to-child HIV prevention programme on infant feeding and caring practices in South Africa. S Afr Med J 2002;92: 298-302.

37 Coutsoudis A: Infant feeding dilemmas created by HIV: South African experiences. J Nutr 2005;135:956-959.

38 Coutsoudis A: Breastfeeding and HIV. Best Pract Res Clin Obstet Gynaecol 2005;19: 185-196.

39 Newell ML, Coovadia H, Cortina-Borja M, et al; Ghent International AIDS Society (IAS) Working Group on HIV Infection in Women and Children: Mortality of infected and uninfected infants born to HIV-infected mothers in Africa: a pooled analysis. Lancet 2004;364:1236-1243.
40 Rollins N, Meda N, Becquet R, et al; Ghent IAS Working Group on HIV in Women and Children: Preventing postnatal transmission of HIV-1 through breastfeeding: modifying infant feeding practices. J Acquir Immune Defic Syndr 2004;35:188-195.

41 Israel-Ballard K, Chantry C, Dewey K, et al: Viral, nutritional and bacterial safety of flash-heated and Pretoria-pasteurized breast milk to prevent mother-to-child transmission of HIV in resource-poor countries: a pilot study. J Acquir Immune Defic Syndr 2005;40:175-181.

42 McDonald C, Curtis H, de Ruiter A, et al: National review of maternity care for women with HIV infection. HIV Med 2006;7:275280.

43 Giles ML, Garland SM, Grover SR, et al: Impact of an education campaign on management in pregnancy of women infected with blood-borne virus. Med J Aust 2006;184: 389-392.

44 Piwoz EG, Ross JB: Use of population-specific infant mortality rates to inform policy decisions regarding HIV and infant feeding. J Nutr 2005;135:1113-1119.

45 De Paoli MM, Manongi R, Klepp KI: Counsellors' perspectives on antenatal HIV testing and infant feeding dilemmas facing women with HIV in northern Tanzania. Reprod Health Matters 2002;10:144-156.

46 Piwoz EG, Ferguson YO, Bentley ME, et al; UNC Project BAN Study Team: Differences between international recommendations on breastfeeding in the presence of HIV and the attitudes and counselling messages of health workers in Lilongwe, Malawi. Int Breastfeed J 2006;1:2.

47 Piwoz EG, Iliff PJ, Tavengwa N, et al: An education and counseling program for preventing breastfeeding associated HIV transmission in Zimbabwe: design and impact on maternal knowledge and behavior. J Nutr 2005;135:950-955.

48 Coutsoudis A, Goga AE, Rollins N, Coovadia HM: Free formula milk for infants of HIV-infected women: blessing or curse? Health Policy Plan 2002;17:154-160.

49 Abiona TC, Onayade AA, Ijadunola KT, et al: Acceptability, feasibility and affordability of infant feeding options for HIV-infected women: a qualitative study in south-west $\mathrm{Ni}$ geria. Matern Child Nutr 2006;2:135-144.

50 Piwoz EG, Ross J, Humphrey J: Human immunodeficiency virus transmission during breastfeeding: knowledge, gaps and challenges for the future. Adv Exp Med Biol 2004; 554:195-210. 